\title{
NUEVAS TECNOLOGIAS EN LOS MECANISMOS ALTERNATIVOS DE SOLUCIÓN DE CONTROVERSIAS PARA EL ACCESO A LA JUSTICIA FRENTE A LOS LÍMITES DEL PODER JURISDICCIONAL
}

\author{
NEW TECHNOLOGIES IN THE ALTERNATIVE MECHANISMS OF \\ DISPUTE SETTLEMENT FOR ACCESS TO JUSTICE AGAINST THE \\ LIMITS OF JURISDICTIONAL POWER
}

\author{
Paola Jackeline Ontiveros Vazquez ${ }^{\mathrm{I}}$ \\ Jesús Manuel Niebla Zatarain ${ }^{\text {II }}$
}

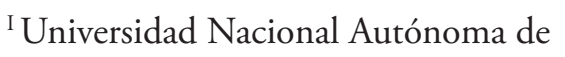
México, Cidade do México, México.

E-mail: paolaontiveros@hotmail.com

II Universidad Autónoma de Sinaloa, Sinaloa, México. Doutor em Direito. E-mail: jmniebla@gmail.com

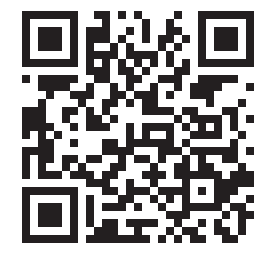

DOI: http://dx.doi.org/10.20912/rdc.v15i37.247

Autores convidados

\begin{abstract}
Resumen: El acceso a la justicia cada vez es más complicado, los órganos jurisdiccionales se encuentran saturados, el tiempo de resolución implica costo, tiempo y desgaste emocional. La tecnología es actualmente una herramienta que nos permite estar en constante comunicación con nuestros seres queridos, realizar negocios desde diferentes partes del mundo y resolver conflictos sin necesidad de trasladarnos; incluir la tecnología en el uso de los mecanismos alternativos de solución de conflictos es un gran avance para la impartición de justicia en México, no solo para descongestionar al sistema judicial, sino para permitir el acceso a la justicia a personas mayores, con capacidades diferentes, que se encuentran fuera de su país de nacimiento o residencia y necesitan el apoyo para resolver un conflicto.
\end{abstract}

Palabras clave: Mecanismos alternativos de solución de controversias. Blockchain. Analítica avanzada. Contratos inteligentes. Inteligencia artificial. Software.

Abstract: Access to justice is becoming more complicated,
jurisdictional structures are saturated, resolution time implies
cost, time and emotional investment. Technology is currently
a tool that allows us to be in constant communication with
our loved ones, have business and negotiations from any
part of the world and resolve conflicts without the need to
transfer form one place to another; Including technology
in the use of alternative dispute resolution mechanisms is a
great advance for the delivery of justice in Mexico, not only
to decongest the judicial system, but to allow access to justice
for older people, people with different capacities or people
who is outside their birth country or residence place and
need support to solve a conflict.

Keywords: Alternative dispute resolution mechanisms,. Blockchain. Cata analytics. Smart contracts. Artificial intelligence. Software. 


\section{Introducción}

Las tecnologías de la información y comunicación (TICs) han replanteado la forma en la cual opera el derecho. El desarrollo y diseminación de entornos digitales, la adopción de procesos que permiten replicar el razonamiento humano a través de tecnología y la masificación de información jurídicamente relevante, son escenarios que requieren un nuevo enfoque jurídico. Como parte de lo anterior, destaca el desarrollo y eventual adopción de plataformas digitales que permitan la resolución de conflictos sin necesidad de la intervención directa de órganos jurisdiccionales, comúnmente conocidos como Métodos Alternativos de Solución de Conflictos (MASCs).

En este sentido, la tecnología ha sido una herramienta indispensable, tanto en la vida de los negocios y en el ámbito jurídico, como en el desarrollo de las relaciones sociales. Permite al ser humano establecer relaciones y producir efectos de derecho sin importar su ubicación geográfica en el mundo. sentirse cercanos, conocer personas, crear negocios, facilitar transacciones transfronterizas, comprar productos, atender quejas y conflictos en línea que permiten la interacción y facilitan la comunicación con los proveedores o individuos involucrados.

No solo se tratará el tema de Alternative Dispute Resolution (ADR), sino también de Online Dispute Resolution (ODR), visto como parte de la revuelta tecnológica 4.0, que marca una revolución cultural y la redefinición de la producción y la economía. Dentro de este contexto es que, todos, los gobiernos, las industrias, las empresas, los abogados, se ven obligados a “...explotar la tecnología digital para mantenerse competitivos en el mercado" 1 .

La utilización de tecnología digital en la resolución de conflictos, no solo se refiere a medios electrónicos, como las computadoras, dispositivos electrónicos, páginas web, videollamadas, incluye elementos tales como como blockchain, inteligencia artificial, smart contracts, analítica avanzada, plataformas digitales que otorguen diversos servicios como negociaciones automatizadas, mediaciones o conciliaciones en línea y procedimientos arbitrales.

Como consecuencia, es importante observar que la tecnología formará parte de los procesos de solución de conflictos; será un elemento indispensable a utilizar para facilitar la comunicación entre personas que no se encuentran en posibilidades de estar en el mismo lugar para una sesión o a las cuales solo les interesa se subsane el daño derivado de un vínculo comercial, sin necesidad de conservar la relación personal entre los involucrados; permitiendo así que se tengan ventajas como la rapidez, eficacia y economía en la resolución de los mismos. De igual forma, permitirá que los órganos

1 Consultado en: http://biblioteca.iiec.unam.mx/index2.php?option=com_content\&do_pdf=1\&id=23226, 3 de mayo de 2019. 
jurisdiccionales reduzcan su carga administrativa, lo que impactará de manera positiva a la impartición de justicia en México.

\section{Mecanismos alternativos de solución de controversias: El entorno}

Actualmente, el uso de la tecnología en los mecanismos alternativos de solución de controversias carece de un marco regulatorio; sin embargo, dichos desarrollos son parte de una realidad en la forma de resolver conflictos. En este sentido, ya existen plataformas digitales como youjustice, Modria, FairClaims, Smartsettle, Mediate, las cuales además ofrecen asistencia de negociación con sugerencias sobre posibles soluciones para ciertos tipos de disputas. En México existen CONCILIANET de la Procuraduría Federal del Consumidor (PROFECO) ${ }^{2}$ y CONDUSEF en línea de la Comisión Nacional para la Protección y Defensa de los Usuarios de Servicios Financieros ${ }^{3}$, donde ya se pueden resolver quejas o problemáticas en línea; otra plataforma que permite llevar a cabo un proceso de mediación en línea y el resguardo del expediente digitalizado en México es Mediación Digital ${ }^{4}$. En lo que respecta a Sudamérica, en Argentina existe un proyecto de mediación comunitaria en línea conocido como MEL ${ }^{5}$; para cuestiones de arbitraje en línea existen servicios como Kluwer Arbitration para resolver cuestiones de arbitraje internacional y sistemas de capacitación en arbitraje en línea que son ofrecidos por la ICC $^{6}$.

Actualmente, el hecho de que no exista una legislación en materia de nuevas tecnologías ${ }^{7}$ enfocadas a los MASCs, es un tema que ha generado polémica aunado a los temores derivados de la adopción de la tecnología en el derecho, los cuales van desde la reducción de la participación del elemento humano en tareas relativas a la impartición de justicia o al diseño de un juez autómata. En síntesis, el enfoque propuesto para el caso específico de los MASCs evita dicha problemática al presentar estos avances como meras extensiones del proceso administrativo de estas, incrementando su eficacia a la parte de una reducción de los recursos que estas requerirían a través de otro tipo de enfoques.

Es así, que "la tecnología (digital) junto con la inteligencia artificial, avanzan, evolucionan y se incorporan más rápidamente en la sociedad y esta los entiende y los absorbe tan fácilmente, que al derecho no le queda otra opción que incorporarlo también,

2 Consultado en: https://concilianet.profeco.gob.mx/Concilianet/inicio.jsp, 5 de agosto de 2019.

3 Consultado en: https://phpapps.condusef.gob.mx/condusefenlinea/TATJ.php, 5 de agosto de 2019.

4 Es una plataforma digital en donde se llevan proceso de mediación para solucionar conflictos. Para más información consultar: https://www.empowerdata.mx/mediacion-digital.html.

5 Para más información consultar: https://www.buenosaires.gob.ar/tramites/mediacion-comunitaria

6 Para más información consultar: https://iccwbo.org/media-wall/news-speeches/icc-unveils-new-onlinetraining-in-international-arbitration/

7 En cuanto a la regulación de servicios financieros mediante el uso e implementación de la tecnología existe en México una Ley para Regular las Instituciones de Tecnología Financiera, conocida como Ley Fintech, publicada en el Diario Oficial de la Federación el 9 de marzo de 2018. Esta ley tiene como finalidad regular empresas emergentes que operen y funcione con sistemas de tecnología financiera. 
pero como todo esto es tan cambiante se va quedando obsoleto y va dejando vacíos jurídicos que más tarde se convierten en problemas graves para la sociedad, como por ejemplo el tema de la privacidad y el uso de los Drones, de la propiedad intelectual de libros, canciones y demás que están colgadas en internet, el tráfico de datos personales en las redes sociales, incluso contratos que las personas aceptan sin saber a cabalidad las consecuencias de los mismos."

Los avances tecnológicos están superando la capacidad regulatoria, lo que provoca la constante actualización en dichos servicios, la implementación de políticas públicas, leyes modelo, previsión normativa, nuevos modelos educativos y la implementación de la misma en la resolución de los conflictos que descongestione a los sistemas judiciales. Al respecto existen tres corrientes de pensadores en relación a la existencia y regularización de nuevas tecnologías?:

- Bioconservadores: Defienden la necesidad de reflexionar sobre los grandes riesgos éticos, jurídicos y políticos, que las tecnologías emergentes pueden generar; buscando mantener la identidad humana y su dignidad, esto es, la conservación de aquello que hace ser un humano. Se oponen al uso de la tecnología para modificar la naturaleza humana, porque causaría una rasgadura de la dignidad humana. Pretenden preservar los atributos individuales que definen al ser humano, conservar al individuo como especie y mantener lo que sería su evolución natural.

- Neurodeterministas: Los seres humanos son singulares conforme a un pensamiento biológico, por tanto el mundo natural y el artificial funcionan según relaciones de causa-efecto donde pueden trabajar armónicamente, y por tanto se maximiza el cerebro manteniendo a la vez la actuación libre de cada ser humano (compatibilistas). Es importante mencionar que por otro lado existen los incompatibilistas en donde consideran que no se pueden mantener a la vez un monismo determinista y la natural libertad.

- Transhumanistas: Término acuñado por el Biólogo J. Huxley, posteriormente usado por Haldane en el siglo XX (1923) ${ }^{10}$, es un estilo de pensamiento que pretende emplear la tecnología para mejorar la vida de cada una de las personas, por ejemplo aumentando la inteligencia y haciendo a los seres humanos más felices y virtuosos, permitiendo una fusión con los seres humanos y las máquinas para la supervivencia y fortalecimiento de las especies.

8 Valencia González, Daniela Alejandra, "Marco jurídico para la inteligencia artificial aplicada a los robots como sistemas autónomos", consultado en: https://repository.usta.edu.co/bitstream/ handle/11634/11686/2017danielavalencia.pdf?sequence $=1$ \&isAllowed $=y$

9 Cfr. De Asis, R. Una mirada a la robótica desde los derechos humanos. Madrid: DYKINSON, S.L Meléndez Valdés, 61 - 28015 Madrid, 2014, págs. 47, 49, 51.

10 El autor analiza una serie de cambios en el desarrollo físico y psicológico del ser humano que conllevaba grandes beneficios y ayudas con el uso de múltiples ciencias y disciplinas como la Ingeniería Genética, la Ingeniería Molecular, La física, La química y otras ciencias destinadas a entender el funcionamiento biológico de los seres humanos. 
Como resultado, la tecnología es percibida desde diferentes conceptos y aplicaciones para el desarrollo del ser humano.

Actualmente, hay debate entre las ventajas y de la complejidad de regular aspectos de la tecnología como la inteligencia artificial, el alcance en la robótica, los sistemas de seguridad y protección de datos, la identificación digital de la persona, el resguardo de la información en la nube, y la inmutabilidad de documentos.

En lo que respecta a los MASCs, en México, son pocos los estados que han contemplado dentro de sus legislaciones la regulación de solución de conflictos empleando los mecanismos alternativos de solución de conflictos a través de medios electrónicos, ya sea con el uso de plataformas digitales, proveedores de videollamadas, servicios de mensajería digital, presentación y resguardo de documentos digitales, etc. Un intento claro es Coahuila donde se regula la presentación de pruebas a través de medios electrónicos para los tribunales arbitrales; el estado de Hidalgo ${ }^{11}$ permite a los interesados hacer uso de los medios electrónicos para resolver un problema a través de los MASCs; en Quintana Roo ${ }^{12}$ las audiencias de procedimientos alternativos se pueden llevar a cabo a través de medios electrónicos; en Tlaxcala ${ }^{13}$ se pueden hacer notificaciones y seguimientos con el uso de medios electrónicos; y en Ciudad de México ya se establece la creación de un Módulo de mediación virtual que permita la prestación del servicio de mediación por medios electrónicos, ópticos o de cualquier otra tecnología ${ }^{14}$, así como la autorización a mediadores privados para prestar el servicio a través de estos medios ${ }^{15}$.

Dichos avances, no han sido del todo considerados en los poderes judiciales, si bien ya existen los juicios en línea en el estado de México ${ }^{16}$, donde ya se reconoce la firma electrónica avanzada y en ningún momento se debe comparecer personalmente ante los juzgados, en otros estados la firma electrónica avanzada no ha sido reconocida para ejercer actos personalísimos ante este tipo de plataformas digitales en la resolución de conflictos.

Se espera en un futuro próximo la aprobación de una Ley General de Mecanismos Alternativos de Solución de Controversias, en la cual se pretende determinar las disposiciones generales para el uso de los MASC a través de medios electrónicos, ópticos o mediante el uso de cualquier otra tecnología, de aprobarse dicha Ley serán responsables los poderes legislativos de cada estado en la adecuación de sus legislaciones para la regulación en dicha materia.

11 Ley de Mecanismos Alternativos de Solución de Controversias para el Estado de Hidalgo, Arts. 17, 18 Y 19.

12 Ley de Justicia Alternativa del Estado de Quintana Roo, Art. 89.

13 Ley de Mecanismos Alternativos de Solución de Controversias del Estado de Tlaxcala, Art. 72.

14 Ley de Justicia Alternativa del Tribunal Superior de Justicia para el Distrito Federal, artículo 15 y Artículo 2, fracción XII Quater.

15 Reglas del Mediador Privado derivados del Acuerdo 24-23/2016 emitido por el Pleno del Consejo de la Judicatura de la Ciudad de México, artículo 22 y artículo 53.

16 Para más información consultar: http://web2.pjedomex.gob.mx/index.php/servicios-en-linea, 1 de agosto de 2019. 


\section{Una forma digital de resolver conflictos}

Actualmente el derecho se encuentra en una era de la transformación donde se hace uso de la tecnología para fomentar una cultura de paz y humanizar los conflictos.

A través de los métodos alternativos de resolución de conflictos en línea, se obtienen ciertas ventajas, tales como: rapidez (las partes y expertos contactan de forma inmediata), eficiencia (todo el procedimiento cuenta con el auxilio complementario de todo un catálogo de recursos TIC), bajo costo (evita o minimiza tanto el transito de personas como el de documentos) y deslocalización (pueden desarrollarse a través de expertos imparciales y resolverse en derecho, en equidad o de forma autocomopositiva, desde cualquier parte del mundo), además se eliminan barreras lingüísticas y geográficas, no solo nacionales, sino aplicables a conflictos transfronterizos. ${ }^{17}$

Las nuevas tecnologías por el momento viables y aplicables en los mecanismos alternativos de solución de controversias para la solución de conflictos en línea (ODR) son: la inteligencia artificial, el blockchain, del cual derivan los smart contracts, y; por último, la analítica avanzada.

El primero, la inteligencia artificial ${ }^{18}$ estudiada en un principio por Alan Turing ${ }^{19}$, como un sistema de modelado de mentes a través de programas de computo. Sin embargo, el concepto ha evolucionado y aunque todavía no se tiene una definición universal, la inteligencia artificial busca entender los procesos cognitivos, la conducta inteligente derivada de la conducta humana, en donde a través de programas informáticos se pueda comprender mejor a la mente. ${ }^{20}$

La inteligencia artificial (IA) permite la resolución de problemas a través de estrategias que emulan el razonamiento humano en un área del conocimiento particular. De esta forma, se tiene la posibilidad de distinguir entre objetos, procesar el lenguaje natural (clasificar, aprender, interpretar el lenguaje humano para generar acciones), responder a ordenes verbales, utilización de reconocimiento de voz (interpretarlas y

17 Vázquez López, Andrés, Realidad virtual y resolución de conflictos en línea, Ed. Alén Media Group, Cordoba, España, 2017, p. 21

18 La IA agrupa un conjunto de técnicas que, mediante circuitos electrónicos y programas avanzados de computadora, busca imitar procedimientos similares a los procesos inductivos y deductivos del cerebro humano. Se basa en la investigación de las redes neuronales humanas y, a partir de ahí, busca copiar electrónicamente el funcionamiento del cerebro. Fuente: http:/www.comoves.unam.mx/numeros/articulo/2/la-inteligenciaartificial-hacia-donde-nos-lleva.

19 La definía como "La disciplina científica y tecnológica que tiene como fin modelar los procesos de la mente y su conexión con el cuerpo a través de la metáfora computacional, es decir mediante programas de cómputo”. Luis Alberto Pineda Cortes, investigador titular del Departamento de Ciencias de la Computación del Instituto de Investigaciones en Matemáticas Aplicadas y en Sistemas de la UNAM. Fuente: http://ciencia.unam.mx/ leer/631/inteligencia-artificial-la-herencia-de-alan-turing-

20 Amador, Hidalgo, Inteligencia Artificial y Sistemas Expertos, Ed. Universidad de Córdoba, España, 1996, pp. $15-20$. 
sacarla a texto o del texto sacarla a voz), identificación de rasgos faciales y emociones, sistemas de planeación que resuelven un negocio, machine learning ${ }^{21}$ y robótica. ${ }^{22}$

La IA aplicada a la resolución de conflictos, tiene como objetivo asistir en el procesamiento de lenguaje para, de esta forma, facilitar dicho servicio a personas que tengan algún tipo de discapacidad visual. Lo anterior, puede aplicarse al acceso de servicios a través de reconocimiento de voz de un comando de voz en particular dentro un proceso de MASC.

Como segundo elemento, se encuentra el blockchain, el cual es una cadena de bloques donde se distribuye información en varios nodos de una red generando un registro público permanente y único, que a su vez, permite transacciones seguras entre usuarios de la misma red, la cual se convierte en inmutable y verificable. ${ }^{23}$ En caso de ser información pública, cualquiera podrá verla y convertirse en nodo; sin embargo la identidad es desconocida, si es información privada, se necesita permiso para convertirse en nodo y debe ser previamente autorizado por una plataforma, existe un administrador central que va a dar las autorizaciones.

En materia de los ODR, el blockchain sirve para proteger el proceso que se lleva a cabo a través de plataformas digitales, permite rastrear su procedencia, es decir cual fue el mecanismo utilizado para resolver el conflicto, cuantas personas intervinieron en el y cual fue el resultado de la negociación, en el caso de ser un convenio o un contrato la información es resguardada a través de esta tecnología que le va a permitir ser un documento inalterable y resguardado por la autoridad correspondiente, además del resguardo de la propiedad intelectual de los documentos y el proceso.

Además, esta herramienta permite la gestión del proceso y el manejo documental, a través del establecimiento de condiciones específicas, dando así transparencia en el proceso de uso de los ODR a través de plataformas digitales, así como llevar un registro de las operaciones realizadas dentro de estos servicios (número de procesos de ODR, en que materia, bajo que mecanismos alternativos, resultados, número de convenios o contratos celebrados, etc.).

El tercer elemento son los smart contracts o contratos inteligentes, derivados del sistema de blockchain que les permite contar con carácter descentralizado, inmutable y transparente; son scripts escritos en lenguaje de programación que permiten ejecutar lo establecido y hacerse cumplir de forma autónoma, automática, sin intermediadores, abogados o mediadores; contienen sentencias y comandos escritos en líneas de código que reemplazan las cláusulas y términos de un contrato tradicional, pueden ser elaborados por

21 Aprendizaje de maquina, base de la inteligencia artificial donde están los algoritmos que permiten las inferencias.

22 Para mas información consultar: http://metrics.digital/estudio-de-madurez-de-la-inteligencia-artificial-enmexico/

23 Definición elaborada en un curso de blockchain con el Ing. Antonio Comesana, noviembre de 2018, en Santiago de Compostela, España. 
personas físicas y/o jurídicas, así como por maquinas o programas automáticos. ${ }^{24}$ Como es posible inferir, la utilización de este tipo tecnología brinda a la par de la ya mencionada seguridad, compatibilidad con plataformas digitales, facilitando de esta manera la accesibilidad a esta plataforma. De igual forma, es capaz de operar con estrategias para el manejo masivo de información digital como lo es big data o la tecnología cloud. El objetivo de los MASCs no es solo la pronta resolución de conflictos, sino operar como una opción de fácil acceso lo cual habrá de generar un alto volumen de información cuya integridad debe estar garantizada por los proveedores de este servicio.

Otra tecnología relevante para los MASCs son los contratos inteligentes, conocidos también como Smart contracts. Existen ciertos beneficios en el uso de esta tecnología, particularmente, cuando una negociación derivada de una mediación genere un convenio en el cual se reflejen determinadas obligaciones y el otorgamiento de derechos concretos. Estos dispositivos no solamente cuentan con validez jurídica, también garantizan la ejecución de las condiciones señaladas por las partes e incluso pueden ser diseñadas para que se adapten en aquellos situaciones donde la consecución del objeto del contrato dependa de variables dinámicas. De manera general, con la adaptación de esta figura, reduciría la posibilidad de incumplimiento por una de las partes minimizando potenciales consecuencias negativas. De igual forma, disminuye la manipulación equivoca o mal intencionada de dicho contrato, dando lugar a una mayor precisión en las transacciones, menor número de intermediarios y tiene un menor $\operatorname{costo}^{25}$.

Por último, la analítica avanzada señala la creación de modelos predictivos, la estadística y la visualización avanzada a conjuntos de datos, procesamiento del lenguaje natural y analítica de texto usando el aprendizaje de maquinas para servir de apoyo a la toma de decisiones en los ámbitos empresariales de cliente, cadena de suministro, finanzas, personal y riesgo. ${ }^{26}$

Esta herramienta, funciona para establecer estadísticas generales sobre la cantidad de procesos que se llevan a cabo en ODR (tipo de conflictos o personas que acuden a un proceso, cantidad de asuntos resueltos, los formatos de convenio que pueden llevar al cumplimiento efectivo de los acuerdos o el número de sesiones necesarias para una mediación familiar), ayuda a establecer patrones de conflictos y las mejores formulas de solución a los mismos, así como las posibilidades de cumplimiento en los acuerdos. Además, puede funcionar como un elemento que ayude a los facilitadores en ODR a encontrar las mejores propuestas de solución o alternativas para un negocio satisfactorio.

24 Consultado en: https://www.alfonsomorant.com/5-beneficios-de-los-smart-contract/, 14 de mayo de 2019.

25 Consultado en: https:/www.alfonsomorant.com/5-beneficios-de-los-smart-contract/. Algunas de las gestiones que se pueden hacer a través de los contratos inteligentes son: transacciones financieras, la gestión de derechos de autor, sistemas de pago, transferencias de derechos y obligaciones, automatización de herencias y donaciones, entre otras, 12 de junio de 2019.

26 Cfr. Consultado en: https://www2.deloitte.com/es/es/pages/technology/solutions/analitica-avanzada.html, 8 de agosto de 2019. 


\section{El ciberespacio y los ODR}

Una de las particularidades de los desarrollos tecnológicos aplicados al campo social, en este caso el derecho, es su compatibilidad con el ciberespacio debido, principalmente a las siguientes características: ${ }^{27}$

- Velocidad de la web

- Disponibilidad de expertos imparciales

- Informalidad y flexibilidad

- Privacidad

- Economía

- Eficiencia

Para atender la naturaleza propia del ciberespacio y la necesidad de contar con dispositivos capaces de razonar, la adopción de agentes inteligentes surge como una propuesta ideal. Esta tecnología es capaz de operar atendiendo requisitos específicos de sus usuarios, detectando (y eventualmente aprendiendo) hábitos particulares para operar acorde a estos, por sus características Negroponte (1995) los denomina mayordomos digitales $^{28}$. En lo que respecta a las propiedades operativas de los agentes Jennings (1995) presenta dos clasificaciones, fuerte y débil. En lo que respecta a la primera, presenta las siguientes propiedades:

- Autonomía: los agentes operan sin intervención directa de operadores humanos y tienen algún tipo de control sobre sus decisiones y su estado interno,

- Habilidad social: agentes pueden operar con otros agentes (e incluso humanos) a través de un lenguaje de comunicación de agentes,

- Reactividad: los agentes perciben el ambiente en el cual operan y responden en tiempo pertinente a los cambios que detectan en este,

- Pro-actividad: agentes no solamente operan reaccionando a su ambiente, son capaces de tomar la iniciativa mostrando comportamiento orientado a objetivos.

- En cambio, aquellos agentes desarrollados bajo el concepto fuerte son sistemas que además de presentar las características de sus contrapartes débiles, son diseñados para implementar conceptos limitados tradicionalmente a humanos, tales como creencia, preferencia, obligaciones, entre otros. Aunado a lo anterior, cuentan con las siguientes propiedades:

- Movilidad: la habilidad de desplazarse a través de redes electrónicas,

- Veracidad: la creencia que un agente no comunicará información falsa,

27 Peruginelli, Ginevra, and Giulia Chiti. "Artificial intelligence in alternative dispute resolution." Proceedings of the Workshop on the law of electronic agents-LEA. 2002.

28 Negroponte, N. (1995), Being Digital, London: Hodder and Stoughton, p. 149-159. 
- Benevolencia: la creencia de que los agentes no tienen objetivos en conflicto y que cada agente, por consecuencia, siempre hará lo que le ha sido ordenado,

- Racionalidad: la creencia de que un agente actuará para conseguir sus objetivos y no actuará de un forma tal que impida el cumplimiento de estos (o por lo menos, mientras no vaya en contra de sus creencias).

ODRs pueden basarse en la tecnología de agentes, debido a que pueden delegar gran parte de las tareas iniciales en estos dispositivos. De tal forma que, en la interacción inicial estos dispositivos podrán recabar información relevante tal como la naturaleza de la disputa e incluso desarrollar propuestas de solución (aunque estas suelen ser meramente resultados de procesos estadísticos y de ninguna manera se equiparan con aquellas desarrolladas por humanos). A su vez asistirán en tareas administrativas, mantendrán a las partes enteradas de fechas relevantes para el proceso, documentación requerida y de otras operaciones propias de la mediación.

\section{Conclusiones}

Las nuevas tecnologías implementadas a los mecanismos alternativos de solución de controversias en línea (ODR) tienen un impacto positivo en sociedad, puesto que incrementan el acceso a la justicia de manera pronta sin depender de procesos jurídicos tradicionales ante órganos jurisdiccionales. De igual forma, esta tecnología permite la inclusión y participación de sectores jurídicos los cuales tenían poca o nula inclusión en estos, como lo es el sector de personas con discapacidad o aquellos los cuales, por su ubicación geográfica les resultaba sumamente difícil desplazarse para acceder a los órganos de impartición de justicia. En este sentido, las ODR han desarrollado un modelo arquitectónico que resulta compatible con el Internet lo que incrementa el sector de usuarios potenciales para esta tecnología.

El uso de las mismas, consiste en un cambio de mentalidad, donde las interacciones entre particulares y empresas, así como las transacciones y la resolución de conflictos se llevan a un nivel diferente de participación, de nuevos conocimientos, de apertura hacia la dinámica interactiva que genera el manejo de la información a través de los sistemas digitales, que permitan lograr mayor porcentaje en cumplimiento y la protección del proceso, como de sus documentos en todo momento. No obstante, el debido aprovechamiento de la tecnología propuesta requiere, en todo momento, de un adecuado nivel de cultura digital de la sociedad a la cual va dirigida. En el caso particular de México, como es el caso en varios países de América Latina, el impacto de las tecnologías de la información en áreas como el derecho se ve limitado considerablemente por la falta de dominio (y en algunos casos familiaridad) con esta tecnología, lo que trae como consecuencia que dichos dispositivos vean limitados su efectividad de manera considerable. 
Para atender lo anterior, el derecho no debe ser reactivo y esperar a que las nuevas tecnologías sean aplicadas de manera aleatoria, o bien conforme a las situaciones se presentan; es momento de tener un derecho proactivo, en donde se pueda generar un marco regulatorio que establezca las condiciones mínimas para el uso de estas tecnologías y el desarrollo de otras nuevas y que fomente, de manera paralela, su entendimiento y uso por parte de la sociedad.

Finalmente, las corrientes de pensamiento en relación a las nuevas tecnologías, establecen los retos que conllevan el uso y desarrollo de estas desde una perspectiva complementaria, alejada de enfoques restrictivos que lo abordan como elementos contrarios a la naturaleza misma del derecho. En consecuencia, estamos ante una nueva era donde las plataformas digitales se presentan como potencializadores del derecho, permitiendo no solamente nuevos enfoques regulatorios, sino soluciones a problemas inherentes al enfoque tradicional de la ley.

\section{Referencias}

Amador, Hidalgo, Inteligencia Artificial y Sistemas Expertos, Ed. Universidad de Córdoba, España, 1996.

De Asis, R. Una mirada a la robótica desde los derechos humanos. Madrid: DYKINSON, S.L Meléndez Valdés, 61 - 28015 Madrid, 2014.

Vázquez López, Andrés, Realidad virtual y resolución de conflictos en linea, Ed. Alén Media Group, Córdoba, España, 2017.

Ley de Mecanismos Alternativos de Solución de Controversias para el Estado de Hidalgo

Ley de Justicia Alternativa del Estado de Quintana Roo

Ley de Mecanismos Alternativos de Solución de Controversias del Estado de Tlaxcala

Ley de Justicia Alternativa del Tribunal Superior de Justicia para el Distrito Federal

Reglas del Mediador Privado derivados del Acuerdo 24-23/2016 emitido por el Pleno del Consejo de la Judicatura de la Ciudad de México.

\section{Fuentes de internet}

Pineda Cortes, Luis Alberto, investigador titular del Departamento de Ciencias de la Computación del Instituto de Investigaciones en Matemáticas Aplicadas y en Sistemas de la UNAM. Fuente: http://ciencia.unam.mx/leer/631/inteligencia-artificial-laherencia-de-alan-turing- 
Valencia González, Daniela Alejandra, "Marco jurídico para la inteligencia artificial aplicada a los robots como sistemas autónomos", consultado en: https:// repository.usta.edu.co/bitstream/handle/11634/11686/2017danielavalencia. pdf?sequence=1\&isAllowed $=y$ http://biblioteca.iiec.unam.mx/index2.php?option=com_content\&do_ $\mathrm{pdf}=1$ \&id=23226, 3 de mayo de 2019.

https://concilianet.profeco.gob.mx/Concilianet/inicio.jsp, 5 de agosto de 2019.

https://phpapps.condusef.gob.mx/condusefenlinea/TATJ.php, 5 de agosto de 2019. https://www.alfonsomorant.com/5-beneficios-de-los-smart-contract/, 14 de mayo de 2019.

https://www2.deloitte.com/es/es/pages/technology/solutions/analitica-avanzada.html, 8 de agosto de 2019. 